\title{
Interpretation of Wall-mounted Probe Characteristics in Hall Thrusters
}

\author{
Rohit Shastry, ${ }^{1}$ Alec D. Gallimore ${ }^{2}$ \\ University of Michigan, Ann Arbor, MI 48109 \\ and \\ Richard R. Hofer ${ }^{3}$ \\ Jet Propulsion Laboratory, California Institute of Technology, Pasadena, CA 91109
}

\begin{abstract}
Wall-mounted Langmuir probes are a promising internal diagnostic for Hall thrusters that can help understand critical plasma-wall interactions. However, the non-ideal environment within the discharge channel can complicate the implementation and interpretation of resulting probe characteristics. The effects of magnetic field and flowing plasma are experimentally investigated on a boron nitride coupon instrumented with a wall-mounted probe and placed in the far-field plume of a Hall thruster. Magnetic field settings were varied from $0-200 \mathrm{G}$, and orientations of $0-90$ degrees were tested. Typical plasma number densities measured were around $3 \times 10^{14} \mathrm{~m}^{-3}$, while electron temperatures were around $1.5 \mathrm{eV}$. The ratio between cyclotron radius and the Debye length ranged from 0.3 to 11, allowing comparisons to be made between this experiment and typical Hall thruster conditions where this ratio ranges from 10-15. It was found that magnetic field and ion flow angle effects were negligible for all orientations except $90^{\circ}$ (parallel to the wall). Based on the results, it is concluded that magnetic fields likely have a negligible effect on measured properties, but the effects of ion flow and magnetic field oriented parallel to the wall requires further investigation.
\end{abstract}

\section{Nomenclature}

$\begin{array}{ll}\lambda_{D} & =\text { Debye length } \\ \lambda_{c} & =\text { cyclotron radius } \\ \varepsilon_{\mathrm{o}} & =\text { permittivity of free space } \\ e & =\text { electron charge } \\ m_{e} & =\text { electron mass }\end{array}$

$\begin{array}{ll}T_{e} & =\text { electron temperature } \\ n_{e} & =\text { plasma number density } \\ v_{t h} & =\text { electron thermal velocity } \\ B & =\text { magnetic field magnitude }\end{array}$

\section{Introduction}

$\mathrm{H}$ all thruster lifetime models currently in development aim to provide predictive tools that would eliminate or substantially reduce the need to perform long-duration, expensive life tests of flight articles [1,2]. A critical part of these modeling efforts is the need to understand and characterize the interaction between the plasma and the discharge channel walls. This interaction is composed of several complex processes that are not fully understood, and yet significantly affect thruster performance and lifetime. Several notable advances have been made in recent years in understanding plasma-wall interactions in Hall thrusters [3-6], but there is presently a noticeable lack of experimental validation. In particular, measurements of ion impact energy on the discharge channel walls would

\footnotetext{
${ }^{1} \mathrm{Ph}$. D. Candidate, Plasmadynamics and Electric Propulsion Laboratory, rshastry@umich.edu, AIAA student member

${ }^{2}$ Arthur F. Thurnau Professor of Aerospace Engineering and Director of the Plasmadynamics and Electric Propulsion Laboratory, alec.gallimore@umich.edu. AIAA Associate Fellow.

${ }^{3}$ Technical Staff Member, Electric Propulsion Group, 4800 Oak Grove Dr., MS 125-109, Pasadena, CA 91109, richard.r.hofer@jpl.nasa.gov. AIAA senior member.
} 
provide an excellent source of validation for current models as well as increase understanding of plasma-wall interactions. However, the complex environment of the Hall thruster sheath complicates the use of standard probes and interpretation of resulting data, warranting investigation into how these probes behave under such conditions.

Langmuir probes mounted within the discharge channel wall have been successfully implemented in the past as an internal diagnostic [7-11]. However, effects of magnetic field, flowing plasma, anisotropy of the electron velocity distribution function, and plasma non-uniformity have not been adequately considered when analyzing data from these probes. These non-ideal features of the plasma within the channel can complicate the obtained results. In order to properly determine ion impact energies within the channel, accurate floating and plasma potentials must be known, and thus the effect of the complex environment on the measured parameters must be determined. This study aims to experimentally characterize the effects of magnetic field and flowing plasma on the characteristics of wall-mounted probes. A boron nitride coupon was instrumented with a flush-mounted Langmuir probe and subjected to various magnetic field configurations using a Helmholtz coil. The coupon was placed within the plume of a 6-kW laboratory Hall thruster, and allowed to rotate in order to investigate various flow and magnetic field orientations. The setup was designed to mimic certain conditions found within the Hall thruster channel, but in a controlled, well-known manner.

The paper is organized as follows: Section II details the experimental apparatus used in this investigation. Section III gives results of the measured plasma properties over the range of configurations tested, while Section IV provides a discussion of the results and its impact on the interpretation of wall-mounted probe characteristics. Finally, Section V draws conclusions from the current work and briefly discusses future work.

\section{Experimental Apparatus}

\section{A. Vacuum Facility}

This study was performed in the Large Vacuum Test Facility (LVTF) at the Plasmadynamics and Electric Propulsion Laboratory. The LVTF is a 6-m wide by $9-\mathrm{m}$ long cylindrical stainless steel chamber. Pumping is provided by seven single-stage CVI model TM-1200 cryopumps, which are covered by liquid-nitrogen-cooled shrouds. Nominal pumping speed is $240,000 \mathrm{l} / \mathrm{s}$ on xenon, providing a base pressure of approximately $2.3 \times 10^{-7}$ torr. Facility pressure was monitored by two commercially available hot-cathode ionization gauges. For a total xenon flow rate of $21.5 \mathrm{mg} / \mathrm{s}$, the operating pressure in the LVTF was $1.2 \times 10^{-5}$ torr.

\section{B. Test Coupon and Langmuir Probes}

The coupon used for this study was a 2.54-cm-diameter disc made of HP grade boron nitride. A 0.5-mmdiameter tungsten wire was inserted at the center of the disc such that its tip was flush with the coupon surface. An alumina post and stainless steel collar was used to connect the probe/coupon assembly to a linear rotational stage. The coupon location was offset slightly from the point of rotation to ensure that the probe location remained fixed during orientation changes (see Figure 1). A separate, cylindrical Langmuir probe was placed $20.5 \mathrm{~cm}$ to the side and $16 \mathrm{~cm}$ in front of the test coupon. This probe allowed for an independent measurement of the local plasma properties not subject to the effects of the imposed magnetic field. The probes were driven by a commercially available Keithley 2410 sourcemeter. Collected current was measured by the same device, and the resulting data were recorded using Labview. 

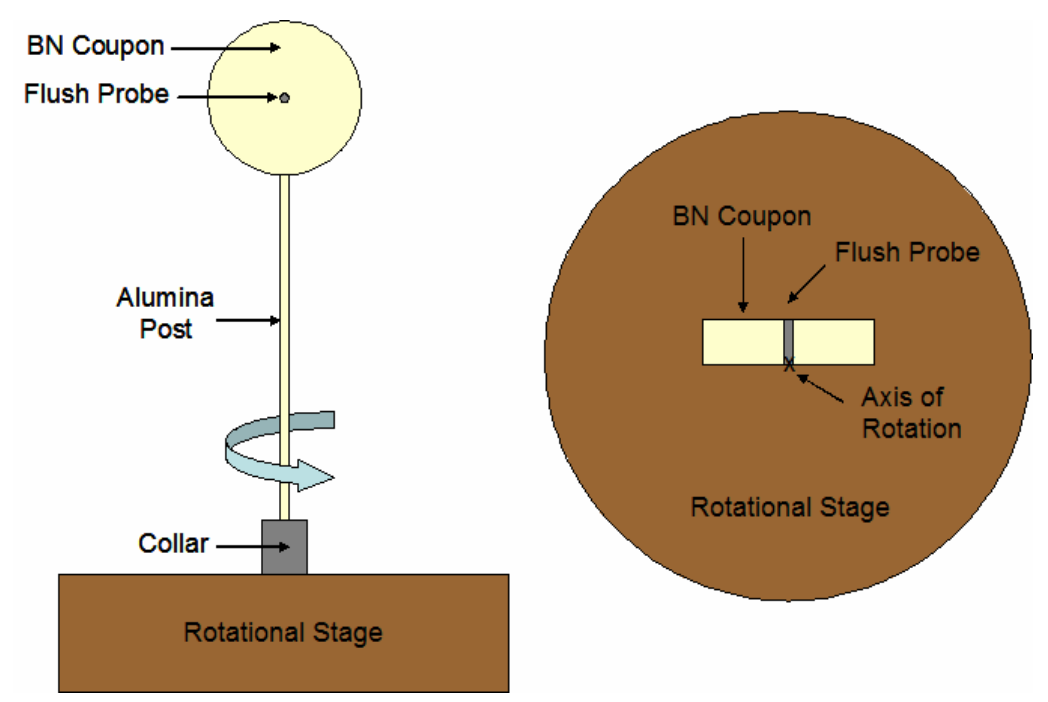

Figure 1: Schematic of coupon/probe assembly used in study. Left: Front view. Right: Top view. Not to scale.

\section{Helmholtz Coil}

The magnetic field for this study was provided by a Helmholtz coil fabricated in-house. Each coil was composed of 200 turns of 13-Gauge magnet wire, wrapped around aluminum spools. Each spool was hollow along the center with an inner diameter of approximately $5 \mathrm{~cm}$. The coils were supported by commercially available aluminum alloy extrusions, and had a separation distance of $18.5 \mathrm{~mm}$. A 350-W commercially available power supply was used to energize the coils, while a digital multimeter placed in series was used to monitor the supplied current.

A 3-axis gaussmeter was used to map the magnetic field along the center plane at several current levels. The ambient magnetic field map was measured and later subtracted out of subsequent data sets. Figure 2 shows the test section of the Helmholtz coil as well as the magnetic field map at 5 A of supplied current. A calibration curve was generated for the magnetic field strength at the center of the test section and used to determine the required current for each data point tested. The magnetic field generated was approximately 26 gauss (G) per ampere (A) at the test section center.
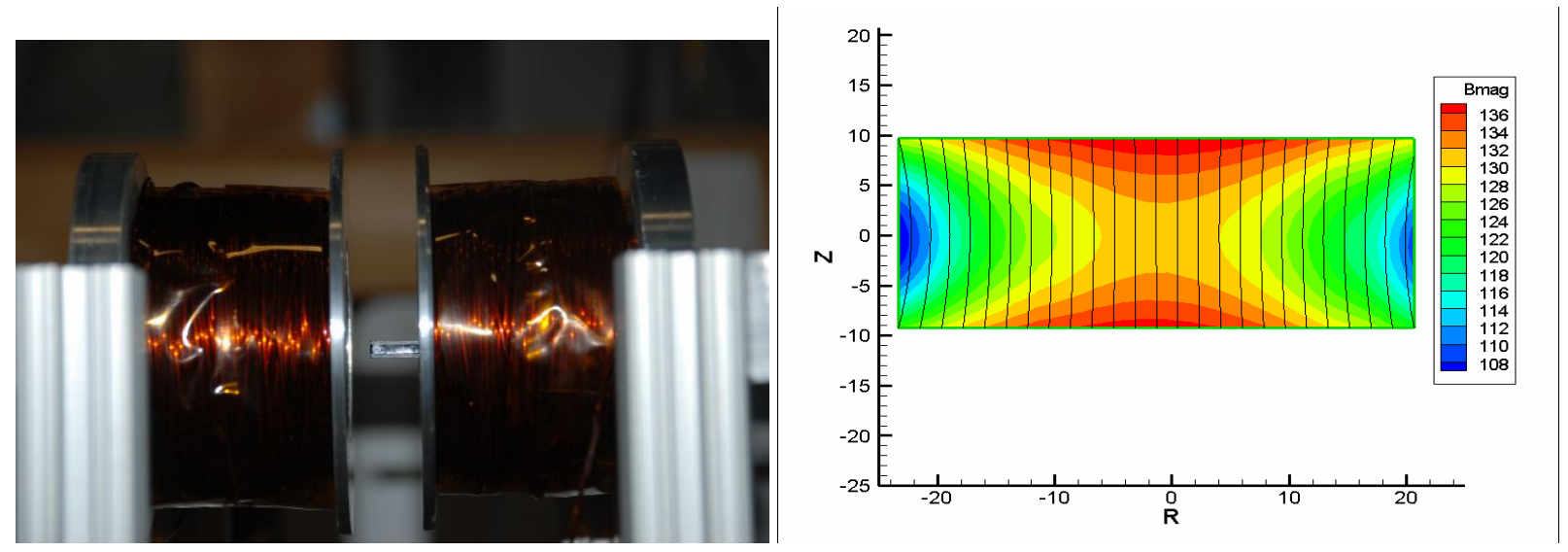

Figure 2: Helmholtz coil used in the experiment. Left: Side view showing gaussmeter in test section. Right: Magnetic field map at $5 \mathrm{~A}$ of supplied current. Units are in gauss, and the origin is the test section center.

\section{Plasma Source}

The Helmholtz coil assembly was placed in the plume of a 6-kW laboratory Hall thruster, approximately $3 \mathrm{~m}$ downstream of the thruster exit and 14 degrees off thruster centerline. Research grade xenon propellant $(99.999 \%$ 
pure) was supplied to the anode and cathode via two separate commercially available flowmeters, accurate to $\pm 1 \%$ full scale. The Helmholtz coil assembly was oriented such that its centerline was aligned with the ion flow, assuming isotropic expansion from a point source located at thruster center (see Figure 3). This was done to ensure that the ion flow and magnetic field were very nearly parallel, such that the plasma flow met little impedance from the magnetic field on its way to the test coupon at the coil center. Uncertainly in magnetic field angle was estimated to be $\pm 1^{\circ}$, while the uncertainty in flow angle was conservatively estimated to be $\pm 5^{\circ}$.

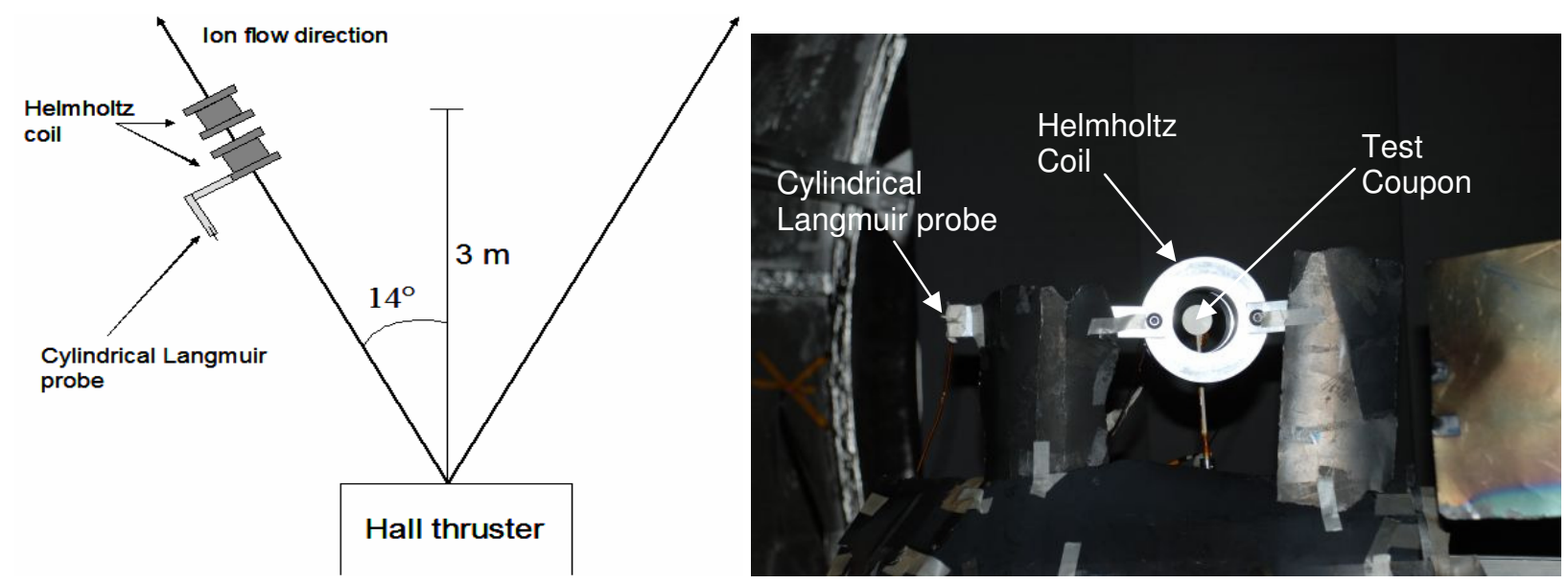

Figure 3: General experimental setup and orientation. Left: Top-down schematic of relative orientation between thruster, Helmholtz coil, and cylindrical Langmuir probe. Not to scale. Right: Front-view photograph showing final integration between test coupon, Helmholtz coil, and cylindrical Langmuir probe.

\section{Results}

Langmuir probe traces were taken under various magnetic field magnitudes and orientations (see Table 1, Figure 4). Since the Helmholtz coil assembly remained fixed with respect to the plume of the Hall thruster, the magnetic field and ion velocity vectors were always parallel. Therefore, differences between the ion flow angle and the magnetic field orientation, a situation that is encountered in Hall thruster discharge channels, were not studied in this experiment. The effects purely from flow angle were investigated by looking at the tested angles with no magnetic field present. Floating and plasma potentials, electron temperature, and number density were found using standard Langmuir probe theory [12], and compared across test conditions. Figure 5 shows the variation in plasma properties over the range of magnetic field and flow angle settings tested.

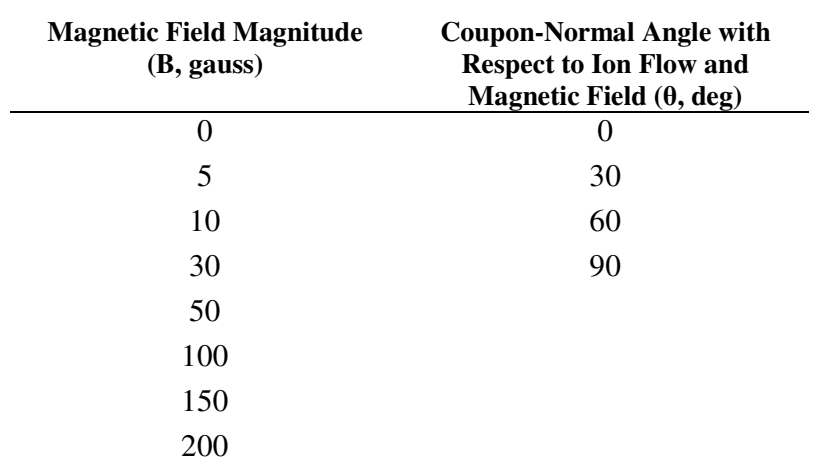

Table 1: List of magnetic field magnitudes and orientation angles tested. All eight magnetic fields were tested at each orientation, yielding a total of 32 test conditions.

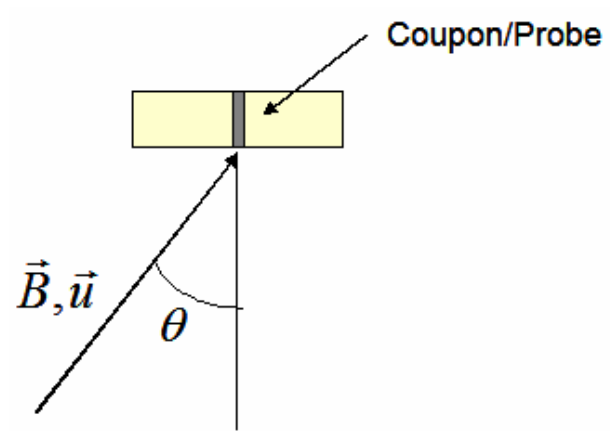

Figure 4: Definition of orientation angle of magnetic field and ion flow with respect to probe. 

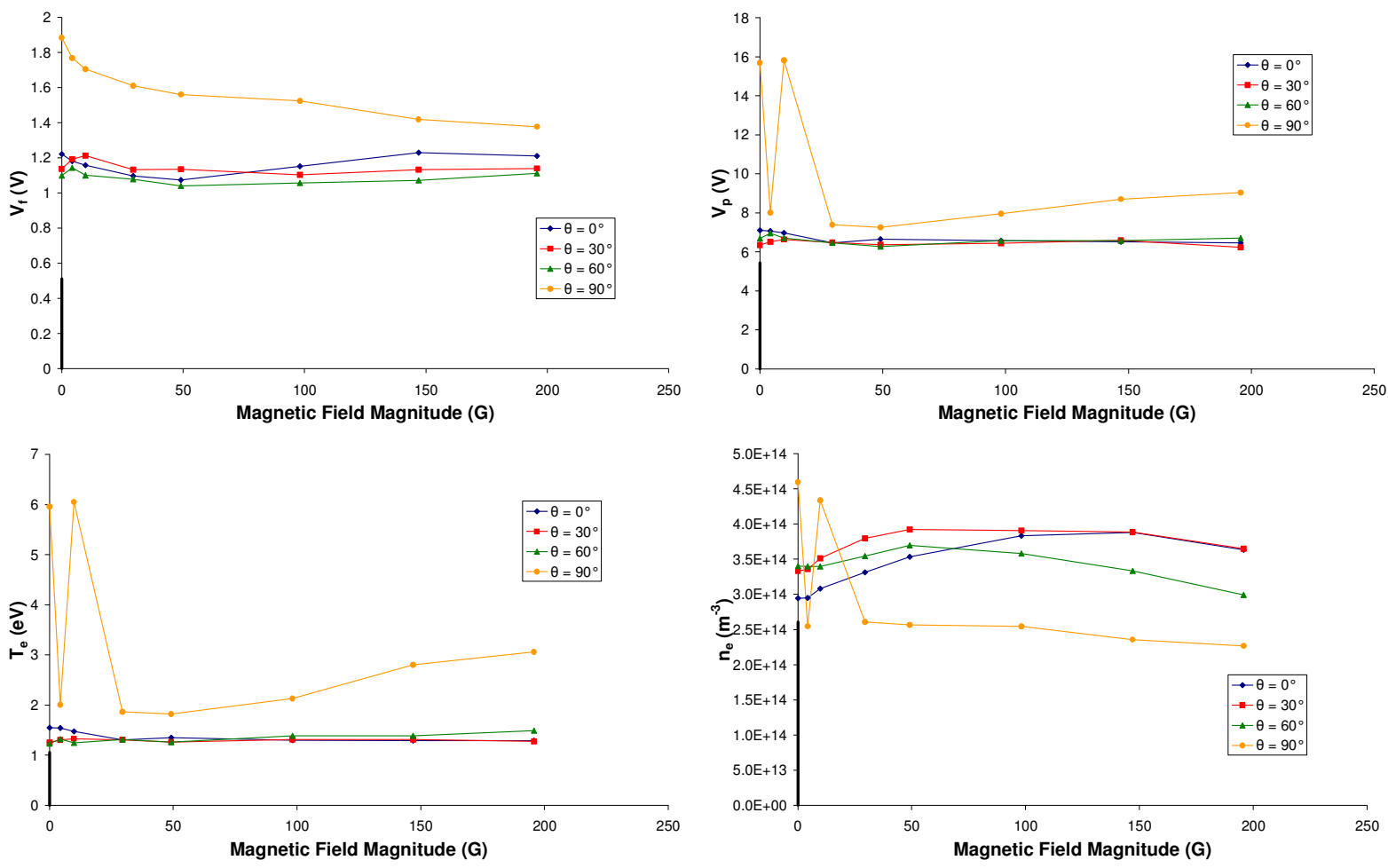

Figure 5: Calculated plasma properties as a function of magnetic field setting. Shown are floating potential (upper left), plasma potential (upper right), electron temperature (lower left), and number density (lower right). The height of the vertical bars at $\mathrm{B}=0$ represents the values measured by the independent cylindrical Langmuir probe.

It is evident from Figure 5 that the effects of flow angle and magnetic field are negligible (i.e. within the experimental error) under the conditions tested for angles less than 90 degrees. The data appear a bit noisy for a 90degree orientation and magnetic field magnitudes less than $25 \mathrm{G}$. For greater magnitudes, the floating potential and number density drop while the plasma potential and electron temperature increase with increasing magnetic field. The independent cylindrical Langmuir probe measured properties of $0.5 \mathrm{~V}$ floating potential, $5.4 \mathrm{~V}$ plasma potential, $1.1 \mathrm{eV}$ electron temperature, and $2.6 \times 10^{14} \mathrm{~m}^{-3}$, all of which are typical of the far-field plume of a Hall thruster (see Figure 5). While all of these properties were measured slightly greater by the coupon probe, the cylindrical probe was farther off thruster centerline and thus more likely to measure lower potentials and densities. This minor discrepancy was therefore not attributed to any inherent difference between cylindrical and flush-mounted probes. Lastly, it should be noted that while the effects of magnetic field on number density at angles less than 90 degrees are small, there are distinct trends. In particular, the number density almost monotonically increases with magnetic field at $\theta=0^{\circ}$, while it tends to peak around $50 \mathrm{G}$ at orientations of 30 and 60 degrees. These features, along with their impact on flush probes in Hall thrusters, will be further discussed in the following section.

\section{Discussion}

The primary goal of this study is to determine the effects of magnetic field and flowing plasma on wall-mounted probes under typical conditions within a Hall thruster discharge channel. In Section III, it was shown experimentally that these effects are small over a wide range of the scenarios tested. In order to better understand this result, one must consider the effects on such a probe as being determined by the resulting changes in the Debye sheath and particle trajectories. In particular, it has been shown by Ahedo [13,14] that the sheath within a magnetic field changes fundamentally based on ratios between the cyclotron radius, Debye length, and collisional mean free path. The mean free path in this experiment as well as in a Hall thruster discharge channel is on the order of a meter, which is much larger than the other two length scales, which are typically less than a millimeter. Therefore, the relevant regime is always collisionless and only the ratio between the cyclotron radius and the Debye length is considered here. Assuming that the ion temperature is negligible, the Debye length is 


$$
\lambda_{D}=\sqrt{\frac{\varepsilon_{o} T_{e}}{e n_{e}}},
$$

where $\varepsilon_{o}$ is the permittivity of free space, $T_{e}$ is the electron temperature in $\mathrm{eV}, e$ is electron charge, and $n_{e}$ is the plasma number density, all in SI units. The cyclotron, or Larmor, radius, is defined as

$$
\lambda_{c}=\frac{m_{e} v_{t h}}{e B}=\frac{m_{e}}{e B} \sqrt{\frac{e T_{e}}{m_{e}}}=\sqrt{\frac{m_{e} T_{e}}{e B^{2}}},
$$

where $m_{e}$ is the electron mass and $B$ is the magnetic field strength in SI units. Combining these equations, the ratio between these lengths is then

$$
\frac{\lambda_{c}}{\lambda_{D}}=\sqrt{\frac{m_{e} n_{e}}{\varepsilon_{o} B^{2}}} .
$$

Table 2 gives values for several relevant quantities near the inner and outer walls of the NASA-173Mv1 [15,16], as well as within this study. Plasma properties within the thruster were measured approximately 0.1 channel widths from each wall [16]. The NASA-173Mv1 was taken as a typical Hall thruster within its power class (several kW). While exact values will vary from thruster to thruster, the magnitude of the values, in particular $\lambda_{d} / \lambda_{D}$, should remain representative of a large class of Hall thrusters.

\begin{tabular}{|c|c|c|c|}
\hline & $\begin{array}{c}\text { Outer Wall } \\
\text { (NASA-173Mv1) }\end{array}$ & $\begin{array}{c}\text { Inner Wall } \\
\text { (NASA-173Mv1) }\end{array}$ & Present Study \\
\hline Number Density $\left(\mathrm{m}^{-3}\right)$ & $7 \times 10^{17}-10^{18}$ & $7 \times 10^{17}-10^{18}$ & $3 \times 10^{14}$ \\
\hline Electron Temperature $(\mathrm{eV})$ & $10-40$ & $10-40$ & 1.5 \\
\hline Cyclotron Radius $(\mu \mathrm{m})$ & $450-650$ & $335-430$ & $145-5840$ \\
\hline Debye length $(\mu \mathrm{m})$ & $30-40$ & $30-40$ & 525 \\
\hline$\lambda_{\mathrm{c}} / \lambda_{\mathrm{D}}$ & $15-16$ & $10-12$ & $0.3-11$ \\
\hline
\end{tabular}

Table 2: Typical values of relevant quantities near the inner and outer walls of the NASA-173Mv1 [15,16], as well as for the present study. Plasma properties for the thruster are based on a $300 \mathrm{~V}$ discharge.

Table 2 shows that $\lambda_{c} / \lambda_{D}$ varies from 0.3 to 11 in this study. Using the measured number density of $2.6 \times 10^{14} \mathrm{~m}^{-3}$, the magnetic field strength required for $\lambda_{d} / \lambda_{D}$ to equal unity is approximately $50 \mathrm{G}$. This indicates that magnetic field magnitudes less than $50 \mathrm{G}$, along with angles between 0 and 60 degrees, represent similar conditions to those found within Hall thruster channels. This regime, where $\lambda_{d} / \lambda_{D} \gg>1$, was shown by Ahedo [13] to consist of a triple layer structure: a magnetized presheath, an unmagnetized Debye sheath, and an intermediate layer known as the Chodura layer. The purpose of the Chodura layer is to turn the plasma from the B-aligned flow in the presheath to the Ealigned flow in the Debye sheath. In this case, it can be argued that since the sheath remains unmagnetized, the effects of the magnetic field on the resulting probe characteristics are small.

For magnetic fields greater than $50 \mathrm{G}$, where $\lambda_{c} \lambda_{D} \sim 1$, Ahedo [13] shows that the sheath is comprised of a doublelayer: a magnetized presheath, and a partially-magnetized Debye sheath. In this particular case, the magnetic field would greatly affect particle trajectories to the probe. However, this was not observed in the experimental data, except in the calculated number density, as well as all plasma properties at the 90-degree orientation. One would expect that within the magnetized sheath, plasma would move towards the probe oriented along the magnetic field lines. Since this would seemingly only affect the capture area of the probe, only the calculated number density would change. As shown in Figure 5, the calculated plasma density noticeably decreases for magnetic fields higher than $50 \mathrm{G}$, as expected if the effective probe area decreases once the sheath becomes magnetized. The increase in number density for magnetic fields less than $50 \mathrm{G}$ can be explained by the plasma being "squeezed" by the magnetic field such that there are more electrons per unit volume. Since there is no effective probe area drop at an orientation of $\theta=0^{\circ}$, no noticeable drop in measured density is observed. 
When $\theta=90^{\circ}$, the magnetic field is parallel (or very nearly parallel) to the coupon and probe surface. This configuration can cause a large amount of magnetic insulation of the incoming electron flow, considerably altering the resulting probe characteristic (see Figure 6). With the exception of anomalous points at 5 and/or $10 \mathrm{G}$, this effect tends to decrease floating potential and number density, while increasing plasma potential and electron temperature.

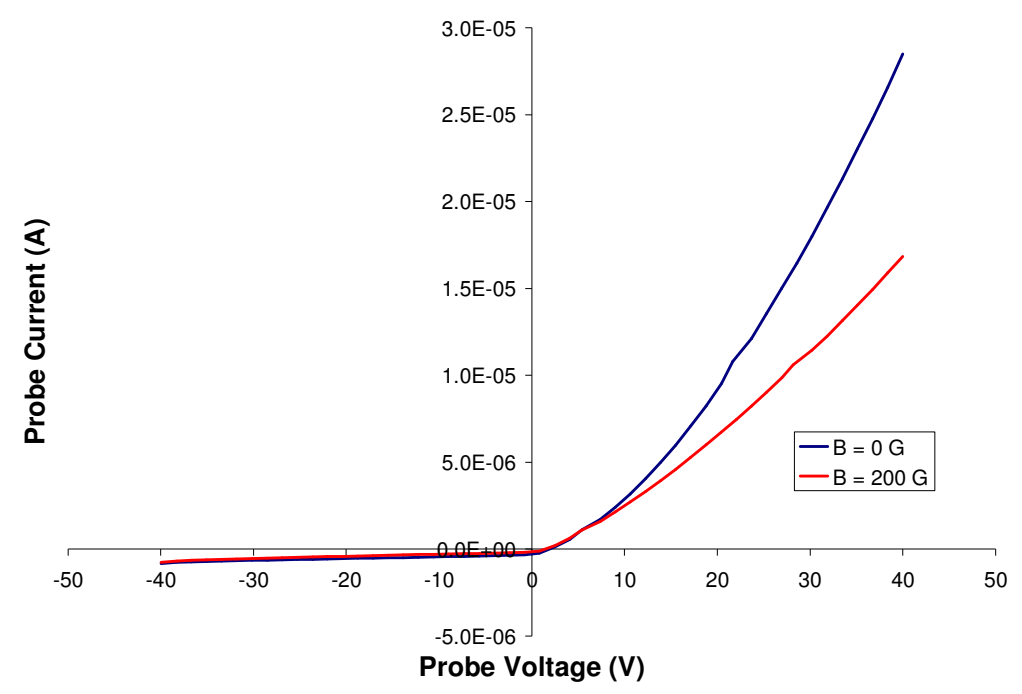

Figure 6: Probe characteristics at $\theta=90^{\circ}$ orientation with 0 and $200 \mathrm{G}$ applied magnetic field strength. Notice the suppression of collected current at $\mathrm{B}=200 \mathrm{G}$.

Figure 6 shows how the magnetic field causes a decrease in the collected current of the probe at a given voltage. This change in shape would artificially flatten the characteristic in the transition regime as well as decrease the measured electron saturation current, causing the observed trends in electron temperature and number density. The reason for the decrease in floating potential, however, is not immediately clear. If one assumes that the ion flux to the probe is largely unaffected by the magnetic field (which is true for unmagnetized ions), then the required electron current to "reach" floating and plasma potentials would require greater probe voltages to overcome the magnetic insulation. This would in turn result in greater measured potentials. However, while the plasma potential measurement follows this trend, the floating potential follows the opposite trend. Thus, the decrease in floating potential at $\theta=90^{\circ}$ requires further investigation.

In regards to the effects of flowing plasma with zero magnetic field, the experimental data shows significant changes in measured properties only when $\theta=90^{\circ}$, i.e. when the flow is parallel to the probe surface. Since the properties presented here are mainly derived from the portion of the probe characteristic where electron current is dominant, it is surprising that the direction of ion flow has such a noticeable effect. This implies that the ion flow direction can affect the electron trajectories enough to alter the measured plasma properties at $\theta=90^{\circ}$. Since this particular configuration will likely be encountered when using wall-mounted probes in Hall thrusters, its effects warrant further investigation.

\section{Conclusions and Future Work}

The effects of magnetic field and flowing plasma on wall-mounted Langmuir probes were experimentally investigated. Under the conditions tested, it was found that the magnetic field and orientation had negligible effects on measured plasma properties for angles less than about 90 degrees. The orientation of ion flow and magnetic field at 90 degrees seemed to have a large effect on all measured properties. Of particular interest were orientations where $\theta$ varied from $0-60^{\circ}$ with magnetic fields less than $50 \mathrm{G}$. These configurations mimic the typical magnetic field orientations at a Hall thruster discharge channel wall, as well as the sheath regime characterized by $\lambda_{c} / \lambda_{D} \gg 1$. Since the measured plasma properties were largely unaffected under these conditions, mainly due to an unmagnetized sheath, it is likely that magnetic field effects will not have to be taken into account when using wall probes in Hall thrusters. However, the addition of electric fields and non-Maxwellian electron distributions, not 
investigated here, may add complexity to the effects of magnetic fields and must be considered. Also, the effects of flowing plasma, in particular when the flow is parallel to the wall, are still relevant to this study, and warrant further investigation to determine whether these effects must be accounted for in implementation and analysis of wall probes.

\section{Acknowledgments}

The authors would like to thank Dan Goebel for fruitful discussion, Ron Watkins for fabrication of the Helmholtz coil used in this study, and Bryan Reid for help in running the plasma source. The authors would also like to acknowledge and thank the Air Force Office of Scientific Research (AFOSR) for funding this research. Dr. Mitat Birkan is the Project Manager for Grant FA9550-06-1-0105.

\section{References}

[1] Hofer, R. R., Mikellides, I. G., Katz, I., and Goebel, D. M., "BPT-4000 Hall Thruster Discharge Chamber Erosion Model Comparison with Qualification Life Test Data," Presented at the 30th International Electric Propulsion Conference, IEPC-2007-267, Florence, Italy, September 17-20, 2007.

[2] Yim, J. T., Keidar, M., and Boyd, I. D., "A Hydrodynamic-Based Erosion Model for Hall Thrusters," Presented at the 29th International Electric Propulsion Conference, IEPC-2005-013, Princeton, New Jersey, October 31 - November 4, 2005.

[3] Ahedo, E. and Parra, F. I., "Partial trapping of secondary-electron emission in a Hall thruster plasma," Physics of Plasmas 12, 7, 073503-073501 - 073503-073507 (2005).

[4] Barral, S., Makowski, K., Peradzynski, Z., Gascon, N., and Dudeck, M., "Wall material effects in stationary plasma thrusters. II. Near-wall and in-wall conductivity," Physics of Plasmas 10, 10, 4137-4152 (2003).

[5] Kaganovich, I., Raitses, Y., and Sydorenko, D., "Electron Kinetic Effects and Beam-Related Instabilities in Hall Thrusters," Presented at the 30th International Electric Propulsion Conference, IEPC-2007-052, Florence, Italy, Sept. 17-20, 2007.

[6] Keidar, M., Boyd, I. D., and Beilis, I. I., "Plasma flow and plasma-wall transition in Hall thruster channel," Physics of Plasmas 8, 12, 5315-5322 (2001).

[7] Azziz, Y., Warner, N. Z., Martinez-Sanchez, M., and Szabo, J. J., "High Voltage Plume Measurements and Internal Probing of the BHT-1000 Hall Thruster," Presented at the 40th AIAA/ASME/SAE/ASEE Joint Propulsion Conference and Exhibit, AIAA-2004-4097, Fort Lauderdale, Florida, July 11-14, 2004.

[8] Kim, V., Grdlichko, D., Kozlov, V., Lazourenko, A., Popov, G. et al., "Local Plasma Parameter Measurements by Nearwall Probes Inside the SPT Accelerating Channel Under Thruster Operation with Kr," Presented at the 38th AIAA/ASME/SAE/ASEE Joint Propulsion Conference and Exhibit, AIAA-2002-4108, Indianapolis, Indiana, July 7$10,2002$.

[9] Kim, V., Kozlov, V., Skrylnikov, A., Sidorenko, E. K., Murashko, V. M. et al., "Investigation of the SPT operation and discharge chamber wall erosion rate under increased discharge voltages," Presented at the 30th International Electric Propulsion Conference, IEPC-2007-151, Florence, Italy, September 17-20, 2007.

[10] Kim, V., Kozlov, V., Skrylnikov, A., Umnitsin, L. N., Svotina, V. V. et al., "Investigation of the Local Plasma Parameters in the SPT Accelerating Channel Under Increased Discharge Voltages," Presented at the 29th International Electric Propulsion Conference, IEPC-2005-004, Princeton, New Jersey, October 31 - November 4, 2005.

[11] Szabo, J. J., Warner, N. Z., and Martinez-Sanchez, M., "Instrumentation and Modeling of a High Isp Hall Thruster," Presented at the 38th AIAA/ASME/SAE/ASEE Joint Propulsion Conference and Exhibit, AIAA-2002-4248, Indianapolis, Indiana, July 7-10, 2002.

[12] Hershkowitz, N., in Plasma Diagnostics: Discharge Parameters and Chemistry, edited by Flamm, D. L. (Academic Press, Inc., 1989), pp. 113-181.

[13] Ahedo, E., "Structure of the plasma-wall interaction in an oblique magnetic field," Physics of Plasmas 4, 12, 4419-4430 (1997).

[14] Ahedo, E., "Plasma-wall interaction in an oblique magnetic field: Model of the space-charge sheath for large potentials and small Debye lengths," Physics of Plasmas 6, 11, 4200-4207 (1999).

[15] Hofer, R. R., "Development and Characterization of High-Efficiency, High-Specific Impulse Xenon Hall Thrusters," Ph.D Dissertation, Aerospace Engineering, The University of Michigan, 2004.

[16] Linnell, J. A., "An Evaluation of Krypton Propellant in Hall Thrusters," Ph.D. Dissertation, Aerospace Engineering, University of Michigan, 2007. 